\title{
Some new examples for nonuniqueness of the evolution problem of harmonic maps
}

\section{Min-Chun Hong}

We find some new examples to show nonuniquence for the heat flow of harmonic maps where weak solutions satisfy the same monotonicity property.

\section{Introduction.}

Let $(M, g)$ be a compact Riemannian manifold (with or without boundary) and let $(N, h)$ be another compact Riemannian manifold without boundary. Let $u$ be a map from $M$ to $N$ which belongs to $H^{1,2}(M, N)$. We define the energy of $u$ by

$$
E(u, M)=\int_{M}|d u|^{2} d M
$$

where $|d u|$ denotes the Hilbert-Schmidt norm of the differential $d u(x)$ (see [EL]). In local coordinates $\left(x^{i}\right)$ and $\left(y^{\alpha}\right)$ on $M$ and $N$, we have

$$
|d u(x)|^{2}=g^{i j}(x) \frac{\partial u^{\alpha}}{\partial x^{i}} \frac{\partial u^{\beta}}{\partial x^{j}} h_{\alpha \beta}(u(x)) .
$$

The map $u:(M, g) \rightarrow(N, h)$ is called a weak harmonic map if $u \in H^{1,2}(M, N)$ and satisfies

$$
\triangle_{M} u+A(u)(d u, d u)=0
$$

in the sense of distributions in $M$ where $\triangle_{M}$ denotes the Laplace-Beltrami operator on $M$ and $A(u)(d u, d u)$ is the second fundamental form of $N$. In local coordinates $\left(x^{i}\right)$ and $\left(y^{\alpha}\right)$ on $M$ and $N$, the harmonic map $u$ satisfies

$$
\tau^{\gamma}(u)=g^{i j}\left(\frac{\partial^{2} u^{\gamma}}{\partial x^{i} \partial x^{j}}-{ }^{M} \Gamma_{i j}^{k} \frac{\partial u^{\gamma}}{\partial x^{k}}+{ }^{N} \Gamma_{\alpha \beta}^{\gamma}(u) \frac{\partial u^{\alpha}}{\partial x^{i}} \frac{\partial u^{\beta}}{\partial x^{j}}\right)=0
$$

where ${ }^{M} \Gamma$ and ${ }^{N} \Gamma$ are the Christoffel symbols of the connections on $M$ and $N$. 
The heat flow for harmonic maps is defined as follows. We say $u(x, t)$ : $M \times[0, \infty) \rightarrow N$ is a weak solution of the following evolution problem:

$$
\begin{aligned}
& \frac{\partial u}{\partial t}=\triangle_{M} u+A(u)(d u, d u) \\
& u(x, 0)=u_{0}(x) \quad \text { for } x \in M \\
& u(x, t)=u_{0}(x) \text { for }(x, t) \in \partial M \times[0, \infty) .
\end{aligned}
$$

The evolution problem for harmonic maps is introduced by Eells and Sampson in a fundamental paper [ES] to prove the existence of smooth harmonic maps in the case that the $N$ has the nonpositive sectional curvature and $u_{0}$ is smooth. Coron and Ghidaglia in [CG] proved that if $M=N=S^{k}$ with $k \geq 3$ the heat flow must blow up for some smooth maps $u_{0}$. Later, Chang, Ding and Ye in [CDY] showed that the heat flow (1.3) must blow up in finite time for some smooth maps $u_{0}$ even for $M=N=S^{2}$. When the dimension of $M$ is 2, Struwe in [S1] proved early the existence and uniqunence of a weak global solution to the heat flow (1.3) where the solution is smooth away from a finite singular point in $M \times[0, \infty)$.

For higher dimensional case; i.e. $\operatorname{dim} M \geq 3$, Chen and Struwe in [CS] (also [CL] and [S2]) proved a global existence of weak solutions of heat flow (1.3) in which the solution is partial regular.

Let $M=B^{3}$ and $N=S^{2}$ where $B^{3}$ and $S^{2}$ denote respectively the unit ball and the unit sphere in $\mathbb{R}^{3}$. In this case, the Problem (1.3) has the following simple form:

$$
\begin{aligned}
& \frac{\partial u}{\partial t}=\Delta u+|\nabla u|^{2} u \\
& u(x, 0)=u_{0}(x) \quad \text { for } x \in B^{3} \\
& u(x, t)=u_{0}(x) \quad \text { for }(x, t) \in \partial B^{3} \times[0, \infty) .
\end{aligned}
$$

Following Chen and Struwe [CS] $u(x, t): B^{3} \times[0, \infty) \rightarrow S^{2}$ is called a weak solution to problem (C) if the $u(x, t)$ satisfies the following (i)-(iv):

(i) $\frac{\partial u}{\partial t} \in L^{2}(M \times[0, \infty)), E((u(\cdot, t)) \leq E(u(\cdot, 0)) \quad \forall t \in[0, \infty)$,

(ii) $u$ satisfies the first equation of (C) in the weak sense on $B^{3} \times(0, \infty)$,

(iii) $u(x, 0)=u_{0}(x)$ in the trace sense,

(iv) $u(x, t)=u_{0}(x)$ on $\partial M \times[0, \infty)$ in the trace sense. 
Coron in $[\mathrm{Co}]$ constructed some examples to show that the heat flow can have infinitely many weak solutions for the same maps $u_{0}$ in the case that $M=\bar{B}^{3}$ and $N=S^{2}$. Coron's idea is to show that the weak solution in [CS](also [CL]) has the following monotonicity property: For any $\theta$ in $C_{0}^{\infty}\left(B^{3}\right)$, any compact $K$ of the interrior of $\{\theta=1\}$ there exists a constant $C$ such that for a. e. $t_{1}, t_{2}$ with $t_{1}<t_{2}$ and for $a$ in $K$

(*) $t_{1}^{-1 / 2} \int_{B^{3}} \theta^{2}|\nabla u|^{2}\left(x, t_{2}-t_{1}\right) \exp -\frac{|x-a|^{2}}{4 t_{1}}$

$$
\leq C\left(t_{2}^{1 / 2}-t_{1}^{1 / 2}\right)+t_{2}^{-1 / 2} \int_{B^{3}} \theta^{2}\left|\nabla u_{0}\right|^{2} \exp -\frac{|x-a|^{2}}{4 t_{2}} .
$$

Then he find some harmonic maps as weak solutions which do not satisfy the above monotonicity property $(*)$. Coron in $[\mathrm{Co}]$ also pointed out that his method does not allow to produce an initial data such that the heat flow has at least two weak solutions satisfiying $(*)$. Then there exists an open problem for the evolution problem of harmonic maps whether weak solutions of heat flow (1.3) with the monotonicity property $(*)$ or the monotonicity inequality for all regular points in [CS] are unique.

In this paper, we give a negative answer to the above problem.

Theorem A. There exist some initial data $u_{0}$ such that the problem (C) has infinitely many weak solutions which satisfy the same monotonicity inequality $(*)$.

Finally, we know from $[\mathrm{F}]$ and [CLL] that all "stationary" weak solutions to Problem (C) also satisfy the energy inequality and the monotonicity inequality defined in [CS] and [CLL], thus all weak solutions in Theorem A satisfy the energy inequality and the monotonicity inequality in regular points as in [CS].

Acknowledgement. The author would like to thank J. F. Grotowski for useful discussions. The work is supported by the Australian Research Council.

\section{The proof of Theorem A.}

Definition 1. A map $u$ is called a weakly stationary harmonic map from $B^{3}$ into the sphere $S^{2}$ if $u$ is a weak harmonic map and also satisfies

$$
\left.\frac{d}{d s} E\left(u_{s} ; B^{3}\right)\right|_{s=0}=0
$$


where $u_{s}(x):=u(x+s \xi(x))$ and $\xi$ has a compact support within $B^{3}$.

Let, for $k$ in $\{1,2,3\}$,

$$
\mu^{k}=\left(u_{x_{j}}^{i} u_{x_{j}}^{i}\right)_{x_{k}}-2\left(u_{x_{k}}^{i} u_{x_{j}}^{i}\right)_{x_{j}} .
$$

We know (see, e. g. [Co], page 341 ) that $u$ is stationary if and only if

$$
\mu^{k}=0 \quad \forall k \in\{1,2,3\} .
$$

Lemma 1. Assume that $u_{0}$ is a weakly stationary harmonic map from $B^{3}$ into $S^{2}$. Then the map $u_{0}$ satisfies the monotonicity inequality $(*)$.

Proof. This results is from the Remark 3 in [Co]. Since $u_{0}$ is a weak stationary harmonic map, $u_{0}$ satisfies (2.1). Then from the proof in [Co], pages $340-341$, we know that $u_{0}$ satisfies the monotonicity inequality ( $*$ ).

Let us recall how a weak solution of (C) is constructed in [CS] (or [CL]). For each $\varepsilon>0$, the Ginzburg-Landau functional $E_{\varepsilon}$ is defined by

$$
E_{\varepsilon}(u):=\frac{1}{2} \int_{B^{3}}\left(|\nabla u|^{2}+\frac{1}{2 \varepsilon^{2}}\left(|u|^{2}-1\right)^{2}\right) d x
$$

for a function $u \in H^{1,2}\left(B^{3}, \mathbb{R}^{3}\right)$. The Euler-Langrange equation is

$$
\begin{aligned}
& \triangle u+\frac{1}{\varepsilon^{2}}\left(1-|u|^{2}\right) u=0, \\
& u(x)=u_{0}(x) \quad \text { for } x \in \partial B^{3}
\end{aligned}
$$

One consider the Cauchy problem for the heat flow associated to $E_{\varepsilon}$

$$
\begin{aligned}
& \frac{\partial u}{\partial t}=\Delta u+\frac{1}{\varepsilon^{2}}\left(1-|u|^{2}\right) u, \\
& u(x, 0)=u_{0}(x) \quad \text { for } x \in B^{3} \\
& u(x, t)=u_{0}(x) \quad \text { for }(x, t) \in \partial B^{3} \times[0, \infty)
\end{aligned}
$$

where $u_{0}$ is given $H^{1,2}\left(B^{3}, S^{2}\right)$. One easily sees that the evolution problem $\left(\mathrm{C}_{\varepsilon}\right)$ has a unique solution $u^{\varepsilon}$ in $C^{0}\left([0, \infty) ; H^{1,2}\left(B^{3}, \mathbb{R}^{3}\right)\right) \cap$ $C^{\infty}\left((0, \infty) \times B^{3} ; \mathbb{R}^{3}\right)$.

Lemma 2. The solution $u^{\varepsilon}(x, t)$ of $\left(\mathrm{C}_{\varepsilon}\right)$ tends to a weak solution $U^{\varepsilon}$ of problem $\left(\mathrm{BD}_{\varepsilon}\right)$ as $t \rightarrow \infty$. 
Lemma 3. (i) There exists a subsequence $\varepsilon_{i}$ such that as $\varepsilon_{i} \rightarrow 0, u^{\varepsilon_{i}}$ tends weakly in $H^{1,2}\left(\bar{B}^{3} \times[0, \infty)\right)$ to a map $\bar{u}(x, t)$ which $u(x, t)$ is a weak solution of $(\mathrm{C})$. Moreover, for a sequence $t_{i}$, the solution $\bar{u}(x, t)$ converges weakly to a weak harmonic map $u_{\infty}(x)$ in $\bar{B}^{3}$ as $t_{i} \rightarrow \infty$.

(ii) The solution $u(x, t)$ of $(\mathrm{C})$ satisfies $(*)$.

Proof. The first part of Lemma 2 is from [CS] and [CL]. The second part of Lemma 2 is from [Co], pages 338-340.

Let us consider a boundary value problem for harmonic maps,

$$
\begin{gathered}
\triangle u+|\nabla u|^{2} u=0 \quad x \in B^{3} \\
u(x)=u_{0}(x)=x \text { on } \partial B^{3} .
\end{gathered}
$$

Lemma 4. For $x_{0} \in \partial B^{3}$, there exists a weak harmonic maps $u_{0}$ to (BD) which is smooth in $\bar{B}^{3} \backslash\left\{x_{0}\right\}$. Moreover, the $u_{0}$ is stationary.

Proof. The existence of the weak harmonic map $u_{0}$, which is smooth $\bar{B}^{3} \backslash\left\{x_{0}\right\}$, is due to Poon in [P]. It is straightful that the $u_{0}$ is stationary since it is smooth inside $B^{3}$ (see, e.g. [GSY]).

Proposition 5. For $\varepsilon>0$, let $U^{\varepsilon}$ be a solution to $\left(\mathrm{BD}_{\varepsilon}\right)$ with $\left.u_{0}\right|_{\partial B^{3}}=x$. The $U^{\varepsilon}$ weakly converges to the minimizing harmonic map $\frac{x}{|x|}$ to (BD) in $H^{1,2}\left(B^{3}, \mathbb{R}^{3}\right)$ as $\varepsilon \rightarrow 0$.

Proof. Using $\left(\mathrm{BD}_{\varepsilon}\right)$, we have the following Pohozaev's identity for $u=U^{\varepsilon}$ :

$$
\begin{aligned}
\sum_{i, j=1}^{3}\left(u_{x_{j}} x_{i} u_{x_{i}}\right)_{x_{j}}= & \sum_{i, j=1}^{3} u_{x_{j} x_{j}} x_{i} u_{x_{i}}+\sum_{j=1}^{3} u_{x_{j}}^{2}+\sum_{i, j=1}^{3} u_{x_{j}} x_{i} u_{x_{i} x_{j}} \\
= & \Delta u \sum_{i=1}^{3} x_{i} u_{x_{i}}+\frac{1}{2} \sum_{i=1}^{3}\left(x_{i}|\nabla u|^{2}\right)_{x_{i}}-\frac{1}{2}|\nabla u|^{2} \\
= & \frac{1}{\varepsilon^{2}} \sum_{i=1}^{3}\left[x_{i}\left(1-|u|^{2}\right)^{2}\right]_{x_{i}}-\frac{3}{4 \varepsilon^{2}}\left(1-|u|^{2}\right)^{2} \\
& +\frac{1}{2} \sum_{i=1}^{3}\left(x_{i}|\nabla u|^{2}\right)_{x_{i}}-\frac{1}{2}|\nabla u|^{2} .
\end{aligned}
$$


Integrating both sides of the above equality gives

$$
\begin{aligned}
\frac{1}{2} \int_{B^{3}}\left|\nabla U^{\varepsilon}\right|^{2} d x & +\frac{1}{2} \int_{\partial B^{3}}\left|\frac{\partial U^{\varepsilon}}{\partial n}\right|^{2} d \sigma \\
& +\int_{B^{3}} \frac{3}{4 \varepsilon^{2}}\left(1-\left|U^{\varepsilon}\right|^{2}\right)^{2}=\frac{1}{2} \int_{\partial B^{3}}\left|\nabla_{\tau} U^{\varepsilon}\right|^{2} d \tau=4 \pi
\end{aligned}
$$

where $n$ is the exterior norm vector of $\partial B^{3}, \nabla_{\tau}$ denote the differential of $u$ on $\partial B^{3}$. From (2.3), there exists a function $V \in H^{1,2}\left(B^{3}, S^{2}\right)$ with $V=x$ on $\partial B^{3}$ such that $U^{\varepsilon_{i}}$ weakly converges in $H^{1,2}\left(B^{3}, \mathbb{R}^{3}\right)$ to a harmonic map $V$ for $\varepsilon_{i} \rightarrow 0$ and

$$
\int_{B^{3}}|\nabla V|^{2} d x \leq \liminf _{\varepsilon_{i} \rightarrow 0} \int_{B^{3}}\left|\nabla U^{\varepsilon_{i}}\right|^{2} d x \leq 8 \pi .
$$

Then from the Theorem in [BCL]; Theorem 7.1, we know that for all map $u \in H^{1,2}\left(B^{3}, S^{2}\right)$ with $\left.u\right|_{\partial B^{3}}=x$,

$$
\int_{B^{3}}|\nabla u|^{2} d x \geq 8 \pi
$$

and $\frac{x}{|x|}$ is the unique minimizing harmonic map from $H^{1,2}\left(B^{3}, S^{2}\right)$. Therefore we know that $V$ must be the map $\frac{x}{|x|}$. Since this is true for any subsequence $\varepsilon_{i} \rightarrow 0, U^{\varepsilon_{i}}$ converges weakly to $\frac{x}{|x|}$ in $H^{1,2}\left(B^{3}, \mathbb{R}^{3}\right)$.

Proof of Theorem A. Let $u_{0}$ be a Poon's harmonic map in Lemma 4. Then we choose $u_{0}$ to be a initial value to the problem (C). Then we know $u_{1}(x, t)=u_{0}(x)$ be a solution to the problem (C) which satisfying $(*)$.

Let us assume that $u^{\varepsilon}(x, t)$ is a solution of $\left(\mathrm{C}_{\varepsilon}\right)$. Then $u^{\varepsilon}(x, t)$ satisfies an energy inequality; i.e. for any $T>0$ and any $\varepsilon>0$,

$$
\int_{0}^{T} \int_{B^{3}}\left|\partial_{t} u^{\varepsilon}\right|^{2} d x d t+\int_{B^{3}}\left|\nabla u^{\varepsilon}(\cdot, T)\right|^{2} d x \leq \int_{B^{3}}\left|\nabla u_{0}\right|^{2} d x
$$

This is easily obatined by choosing a test function $\partial u^{\varepsilon}$ in $\left(\mathrm{C}_{\varepsilon}\right)$ (see [CS]). By Lemma 3 , there exists a subsequence $\varepsilon_{k}$ such that as $k \rightarrow \infty, u^{\varepsilon_{k}}(x, t)$ weakly converges to $u(x, t)$ in $H^{1,2}\left(B^{3}, \mathbb{R}^{3}\right)$ for $t>0$ where $u(x, t)$ is a solution of heat flow (C) by Lemma 3 and $u(x, t)=x$ on $\partial B^{3}$. From (2.5), we have for any $T>0$

$$
\limsup _{k \rightarrow \infty} \int_{0}^{T} \int_{B^{3}}\left|\partial_{t} u^{\varepsilon_{k}}\right|^{2} d x d t \leq \int_{B^{3}}\left|\nabla u_{0}\right|^{2} d x
$$


Then by the Fatou lemma, we know

$$
\int_{0}^{T} \liminf _{k \rightarrow \infty} \int_{B^{3}}\left|\partial_{t} u^{\varepsilon_{k}}\right|^{2} d x d t \leq \int_{B^{3}}\left|\nabla u_{0}\right|^{2} d x .
$$

Letting $T \rightarrow \infty$ in (2.7), we have

$$
\int_{0}^{\infty} \liminf _{k \rightarrow \infty} \int_{B^{3}}\left|\partial_{t} u^{\varepsilon_{k}}\right|^{2} d x d t \leq \int_{B^{3}}\left|\nabla u_{0}\right|^{2} d x
$$

From (2.8), there exists a subsequence $t_{i}$ such that as $t_{i} \rightarrow \infty$,

$$
\lim _{t_{i} \rightarrow \infty} \int_{t_{i}-1}^{t_{i}} \liminf _{k \rightarrow \infty} \int_{B^{3}}\left|\partial_{t} u^{\varepsilon_{k}}\right|^{2} d x d t=0
$$

By Hölder's inequality, energy inequality (2.5) and the above identity, we have

$$
\lim _{t_{i} \rightarrow \infty} \int_{t_{i}-1}^{t_{i}} \liminf _{k \rightarrow \infty} \int_{B^{3}}\left|\partial_{t} u^{\varepsilon_{k}}\right|\left|\nabla u^{\varepsilon_{k}}\right| d x d t=0
$$

On the other hand, using $\left(\mathrm{C}_{\varepsilon}\right)$, we have the following Pohozaev's identity for $u=u^{\varepsilon_{k}}(x, t)$ :

$$
\begin{aligned}
\sum_{i, j=1}^{3}\left(u_{x_{j}} x_{i} u_{x_{i}}\right)_{x_{j}}= & \sum_{i, j=1}^{3} u_{x_{j} x_{j}} x_{i} u_{x_{i}}+\sum_{j=1}^{3} u_{x_{j}}^{2}+\sum_{i, j=1}^{3} u_{x_{j}} x_{i} u_{x_{i} x_{j}} \\
= & \Delta u \sum_{i=1}^{3} x_{i} u_{x_{i}}+\frac{1}{2} \sum_{i=1}^{3}\left(x_{i}|\nabla u|^{2}\right)_{x_{i}}-\frac{1}{2}|\nabla u|^{2} \\
= & \frac{1}{\varepsilon^{2}} \sum_{i=1}^{3}\left[x_{i}\left(1-|u|^{2}\right)^{2}\right]_{x_{i}}-\frac{3}{4 \varepsilon^{2}}\left(1-|u|^{2}\right)^{2} \\
& +\frac{1}{2} \sum_{i=1}^{3}\left(x_{i}|\nabla u|^{2}\right)_{x_{i}}-\frac{1}{2}|\nabla u|^{2}+\partial_{t} u \sum_{i=1}^{3} x_{i} u_{x_{i}} .
\end{aligned}
$$

Integrating both sides of the above equality (Note $u=u^{\varepsilon_{k}}(x, t)$ ) gives

$$
\begin{aligned}
& \frac{1}{2} \int_{B^{3}}\left|\nabla u^{\varepsilon_{k}}(\cdot, t)\right|^{2} d x+\frac{1}{2} \int_{\partial B^{3}}\left|\frac{\partial u^{\varepsilon_{k}}(\cdot, t)}{\partial n}\right|^{2} d \sigma+\int_{B^{3}} \frac{3}{4 \varepsilon^{2}}\left(1-\left|u^{\varepsilon_{k}}(\cdot, t)\right|^{2}\right)^{2} \\
& \quad=\frac{1}{2} \int_{\partial B^{3}}\left|\nabla_{\tau} u^{\varepsilon_{k}}\left(\cdot, t_{k}\right)\right|^{2} d \sigma+\int_{B^{3}} \partial_{t} u^{\varepsilon_{k}}(\cdot, t) \sum x_{i} u_{x_{k}}^{\varepsilon_{k}}(\cdot, t) d x \\
& \quad=4 \pi+\int_{B^{3}} \partial_{t} u^{\varepsilon_{k}}(\cdot, t) \sum x_{i} u_{x_{k}}^{\varepsilon_{k}}(\cdot, t) d x
\end{aligned}
$$


where $n$ is the exterior norm vector of $\partial B^{3}, \nabla_{\tau}$ denote the differential of $u$ on $\partial B^{3}$.

Next, we will prove the the heat flow (C) has at lest two different solutions by a contradicted method, i.e. assume the weak solutions of heat flow (C) are unique, i.e. $u(x, t) \equiv u_{0}$ (otherwise, weak solutions has at least two solutions). Letting $\varepsilon_{k} \rightarrow 0$ in (2.10),

$$
\begin{aligned}
\int_{B^{3}}\left|\nabla u_{0}\right|^{2} d x & \leq \liminf _{k \rightarrow \infty} \int_{B^{3}}\left|\nabla u^{\varepsilon_{k}}(\cdot, t)\right|^{2} d x \\
& \leq 8 \pi+C \liminf _{k \rightarrow \infty} \int_{B^{3}}\left|\partial_{t} u^{\varepsilon_{k}}\right|\left|\nabla u^{\varepsilon_{k}}\right| d x d t
\end{aligned}
$$

Intergrating (2.11) on $\left[t_{i}-1, t_{i}\right]$, letting $t_{i} \rightarrow \infty$ and using (2.9) gives

$$
\begin{aligned}
\int_{B^{3}}\left|\nabla u_{0}\right|^{2} d x & \leq 8 \pi+C \lim _{t_{i} \rightarrow \infty} \int_{t_{i}-1}^{t_{i}} \liminf _{k \rightarrow \infty} \int_{B^{3}}\left|\partial_{t} u^{\varepsilon_{k}}\right|\left|\nabla u^{\varepsilon_{k}}\right| d x d t \\
& =8 \pi
\end{aligned}
$$

since $u_{0}$ does not depends on $t$.

From the Theorem in [BCL]; Theorem 7.1, we know that for all map $u \in H^{1,2}\left(B^{3}, S^{2}\right)$ with $\left.u\right|_{\partial B^{3}}=x$,

$$
\int_{B^{3}}|\nabla u|^{2} d x \geq 8 \pi
$$

and $\frac{x}{|x|}$ is the unique minimizing harmonic map from $H^{1,2}\left(B^{3}, S^{2}\right)$, so we know that $u_{0}$ must be the minimizing harmonic map $\frac{x}{|x|}$, this is contradicted by our initial condition. This means that the solution $u(x, t)$ is different from $u_{1}(x, t)=u_{0}(x)$ This proves that the problem (C) has two different solutions satisfying $(*)$. Infinitely many solutions can be easily proven by the same steps in $[\mathrm{Co}]$.

From the proof of Theorem A, we have

Corollary. Aussume that $u_{0}: B^{3} \rightarrow S^{2}$ be a non-minimizing weak harmonic map with the boundary condition $u_{0}(x)=x$ on $\partial B^{3}$. Choosing the $u_{0}$ as a initial data to the problem (C), then the problem (C) has infinitely many weakly solutions.

Remark. From the result in $[\mathrm{P}]$, there exists a non-minimizing weak harmonic $u$ with the boundary condition $u(x)=x$ on $\partial B^{3}$ such that $u \in C^{\infty}\left(\bar{B}^{3} \backslash\left\{x_{0}\right\}\right)$ for any $x_{0} \neq 0 \in B^{3}$. 


\section{References.}

[BCL] H. Brezis, J.-M. Coron and E.H. Lieb, Harmonic maps with defects, Commun. Math. Phys. 107 (1986), 649-705.

[CDY] K.-C. Chang, W.-Y. Ding, and R. Ye, Finite-time blow up of the heat flow of harmonic maps from surfaces, J. Diff. Geom. 36 (1992), 507-515.

[CLL] Y. Chen, J. Li and F.-H. Lin, Partial regularity for weak heat flows into sphere, Comm. on Pure and Applied math. 48 (1995), 429-448.

[CL] Y. Chen and F.-H. Lin, Evolution of harmonic maps with Dirichlet boundary conditions, Comm. in Anal. and Geometry 1 (1993), 327-346.

[CS] Y. Chen and M. Struwe, Existence and partial regular results for the heat flow for harmonic maps, Math. Z. 201 (1989), 83-103.

[Co] J.-M. Coron, Nonuniqueness for the heat flow of harmonic maps, Ann. Inst. Henri Poincaré, Analyse non linéaire 7 (1990), 335-344.

[CG] J.-M. Coron and J. M. Ghidaglia, Explosion en tems fini pour le flot des applicationsharmoniques, C. R. Acad. Sci., Paris 308 (1989), 339-344.

[EL] J. Eells and L. Lemaire, Another report on Harmonic maps, Bull. London Math. Soc. 20 (1988), 385-524.

[ES] J. Eells and J. H. Sampson, Harmonic mappings of Riemannian manifolds, Amer. J. Math. 86 (1964), 109-160.

[F] M. Feldman, Partial regularity for harmonic maps of evolution into sphere, Comm. PDES 19 (1994), 761-790.

[GSY] J. F. Grotowski, Y. Shen and S. Yan, On various classes of harmonic maps, Arch. Math. 64 (1995), 353-358.

[P] C.-C. Poon, Some new harmonic maps from $B^{3}$ to $S^{2}$, J. Diff. Geom. 34 (1991), 165-168.

[S1] M. Struwe, On the evolution of Harmonic maps of Riemannian surfaces, Commun. Math. Helv. 60 (1985), 558-581.

[S2] M. Struwe, On the evolution of harmonic maps in higher dimensions, J. Diff. Geom. 28 (1988), 485-502.

RECEIVED November 27, 1996. 
CENTRE FOR MATHEMATICS AND ITS APPLICATIONS The Australian national UnIVERsity

ACT 0200, Canberra, Australia 